\title{
Le macrobenthos de la Saône
}

\author{
H. Tachet ${ }^{2}$ \\ O. Gaschignard-Fossati2 \\ B. Cellot ${ }^{2}$ \\ A. Berly2
}

Mots clés : Grands cours d'eau, Saône (rivière), macrobenthos, zonation longitudinale.

Une série de dragages effectués dans la Saône au niveau de 13 stations réparties sur $377 \mathrm{~km}$ de rivière montre que le macrobenthos du chenal de cette rivière est, dans les stations les moins dégradées, largement dominé par les Ephémérop. teres fouisseurs (Ephoron virgo et Ephemera lineata). La comparaison avec le macrobenthos du Haut-Rhône indique que ce demier, qui présente des caractéristiques mésologiques et hydrologiques similaires, se sittia typologiquement plus à l'amont que la Saóne. La durée et l'importance de l'étiage combinées avec les différences de pente, cette demière influen. çant la vitesse du courant et la granulométrie, sont probablement les facteurs essentiels qui structurent les peuplements macrobenthiques de ces deux cours d'eau.

The macrobenthos of the Saone river

Keywords : large rivers, Saone river, macrobenthos, longitudinal zonation.

The macrobenthos of the Saône river (France) has been sampled with a dredge at 13 stations distributed along $377 \mathrm{~km}$. The macrobenthos of this large river is dominated, in the least altered stations, by the burrowing may flies/Ephoron virgo and Ephemera lineata). The comparison of these benthic communities with those of the Upper Rhone river, a water course with similar discharges and dimensions, shows that the Saone is, typologically, more downstream than the Upper Rhône. The importance and the duration of low waters combined with the differences of slope are probably the most significant factors defining the composition of benthic communities of these two rivers.

\section{Introduction}

"Flumen est Arar,..., incredibili lenitate, ita ut oculis in utram partem fluat judicari non possit". C'est par ce commentaire de Caesar que la Saône (Arar) fait son entrée dans l'Histoire. En dépit de l'ancienneté de cette citation, soulignant la lenteur excep. tionnelle de cette rivière, nos connaissances sur les communautés benthiques de la Saône et plus particulièrement son macrobenthos sont extrèmement réduites. En effet, à l'exception des travaux de Paris (1931) consacrés aux Cladocéres et ceux de Denis et al. (1936) et de Fontaine (1959) concernant les chu. tes de " mannes ", aucun travail n'a été publié avant

1. Structure et fonctionnement des écosystèmes du Haut-Rhóne françats: 52 .

2. UA C.N.R.S. 367, Ecologie des Eaux, Université Lyon I, 43 Bd

11 Novembre 1918, F-69622 Villeurbanne cédex. celui de Régis et al. (1980). Récemment, plusieurs études, non encore publiées, ont été réalisées par Bourrain \& Bourrain (1983) et le CEMAGREF (1984). L'Agence de Bassin Rhóne-Méditerranée-Corse (1984) a publié une mise au point sur la qualité des eaux de la Saône, reprenant, pour l'aspect biologique, une partie des résultats des travaux cités cidessus. Le travajl de Leynaud et al. (1972), consacré à l'ensemble de la Saône, ne concernait que la qualité physico-chimique de ses eaux. Une étude sur l'application des indices biotiques aux grands cours d'eau, dont la Saône, vient d'être publiée par Cellot et al. (1987).

La majorité de ces travaux est basée sur des résultats obtenus à partir de relevés effectués au niveau des berges ou des herbiers, c'est-à-dire en eau peu profonde. Or dans la Saône, dont la largeur moyenne est de 150 mètres et la profondeur moyenne de 
5 mètres, de telles zones n'occupent, en fait, qu'une position très marginale et représentent à peine $7 \%$ des surfaces colonisables par les macroinvertébrés, la majeure partie du lit de la rivière étant recouverte par une lame d'eau de plusieurs mètres d'épaisseur. Le présent travail est donc consacré au macrobenthos du chenal de la Saône. Il repose sur l'analyse d'une série de prélèvements effectués en 1983, par dragage, au niveau de 13 stations réparties sur 377 kilomètres de rivière. Nous examinerons d'abord l'évolution longitudinale des peuplements macrobenthiques en fonction de différentes composantes naturelles ou artificielles susceptibles de les modifier. Nous étudierons ensuite la structure globale de ces peuplements et, par une comparaison avec ceux du Haut-Rhône, nous essaierons de définir les caractéristiques typologiques des peuplements macrobenthiques de la Saône de manière à déterminer ce qui reste des peuplements originels.

\section{La Saône}

\subsection{Le millieu naturel}

La Saône prend sa source au pied des Vosges à 405 m d'altitude ( $f i g .1$ et 2 ) et draine un bassin versant d'environ $29900 \mathrm{~km}^{2}$. Elle rejoint le Rhône à Lyon (altitude 160,5 mètres) après un parcours de $482 \mathrm{~km}$ (Parde 1925). Son affluent principal est le Doubs dont la longueur est de $400 \mathrm{~km}$. Excepté pendant les 65 premiers kilomètres, la Saône coule sur une plaine alluviale constituée de sédiments tertiaires qui correspondent au comblement du Fossé Bressan. Les pentes sont faibles (en général 0,1 à $0,2 \mathrm{~m} / \mathrm{km}$ ) voire très faibles, seulement $0,04 \mathrm{~m} / \mathrm{km}$ sur $132 \mathrm{~km}$ après sa confluence avec le Doubs. Les pentes de ce dernier sont en général plus fortes : de $0,3 \mathrm{~m}$ à $0,5 \mathrm{~m} / \mathrm{km}$ depuis Besançon jusqu'à la confluence. Les pentes du Haut-Rhône (entre Seyssel et Lyon), avant sa confluence avec la Saône, sont de l'ordre de $0,55 \mathrm{~m} / \mathrm{km}$ (Perrin 1978).

\subsection{Les aménagements}

La Saône, comme tous les grands cours d'eau de plaine, a subi divers aménagements destinés surtout à limiter les dégâts liés aux inondations et à faciliter la navigation.

Rivière lente, la Saône est redoutée pour l'ampleur de ses inondations (sa largeur peut dépasser deux kilomètres pendant les crues hivernales). Malgré la construction de digues, les risques liés aux inondations n'ont pas été éliminés (fig. 3a) et, excepté quelques villes comme Chalon, Mâcon et Villefranche, les rives de la Saône sont en général peu urbanisées, prairies et peupleraies occupant la majeure partie des zones inondables.

De nombreux méandres ont été recoupés pour faciliter la navigation (la longueur navigable passant de 407 à 363 kilomètres), mais ces portions courtcircuitées ( $f$ ig. 3 b) diffèrent peu hydrologiquement des autres secteurs parce que les barrages édifiés à l'amont laissent passer l'eau au moment des crues et parce que la largeur des dérivations étant de l'ordre d'une vingtaine de mètres, la majeure partie du débit passe par les parties court-circuitées. Plusieurs barrages-écluses ont, par ailleurs, été édifiés pour relever le niveau de l'eau et éviter ainsi l'arrêt de la navigation pendant l'étiage. Quel que soit leur type, tous ces barrages laissent passer l'eau au moment des crues. De ce fait, il n'y a pas accumulation de sédiments à l'amont des barrages comme cela se produit pour les barrages de retenue.

Cinq canaux relient la Saône à d'autres réseaux hydrographiques (fig. 1). Ce sont de l'armont vers I'aval : le canal de l'Est (liaison avec la Moselle), le canal de la Marne à la Saône, le canal du Rhône au Rhin, le Canal de Bourgogne (liaison avec la Seine) et le canal du Centre (liaison avec la Loire). Les canaux arrivant en rive droite sont plus anciens (fin du XVIII' siècle) que ceux qui arrivent en rive gauche (milieu du XIX). Ces canaux ont permis l'arrivée dans la Saône de nouvelles espèces comme par exemple Dreissenta polymorpha vers $1850-1860$ (Locard 1893) ou plus récemment Gammarus roseli (Pacaud 1952; Jazdzewski \& Roux 1987).

\subsection{Caractéristiques mésologiques fondamentales}

\section{GRANULOMÉtrie}

Pour comparer les granulométries du Rhône et de la Saône, deux prélèvements de $10 \mathrm{~kg}$ ont été réalisés par dragage, au niveau du chenal, l'un dans la Saône à Beauregard, un peu en amont de Villefran. che et l'autre dans le Rhône au niveau de Jons. Les sédiments ont été tamisés sur une série de tamis dont le plus fin était constitué par un tamis de $250 \mu \mathrm{m}$. Les résultats de cette analyse sont représentés sur la figure 4 . Les sédiments du Haut-Rhône 


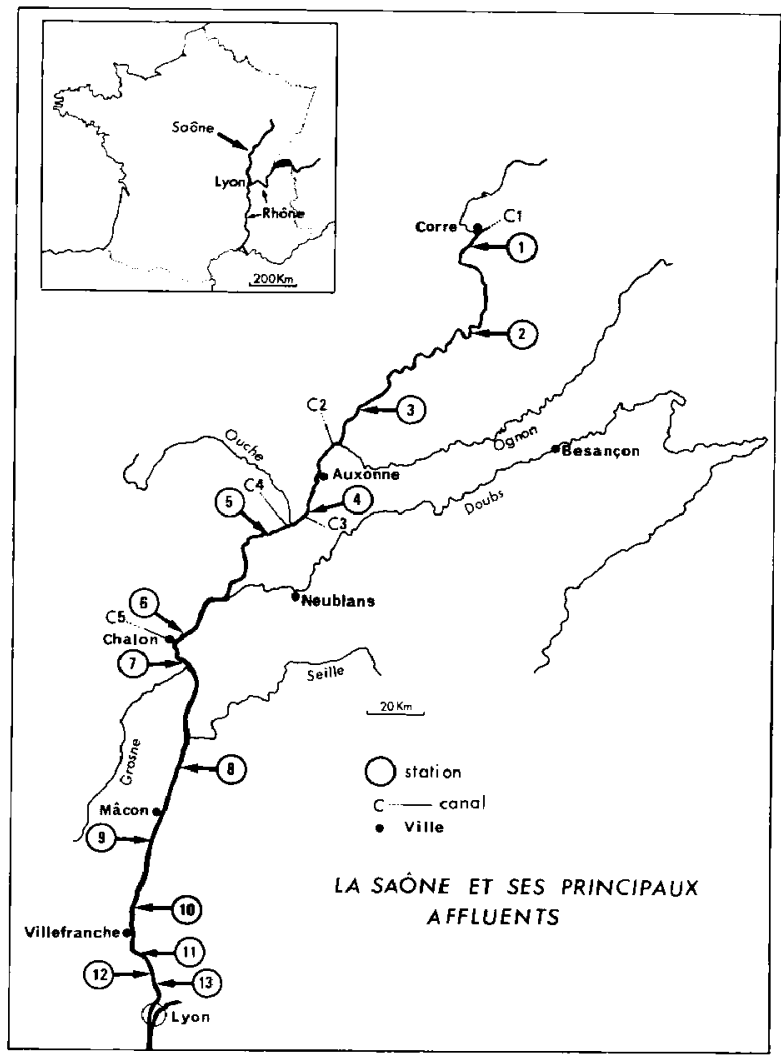

Fig. 1. Carte de la Saóne et de ses principaux affluents

Cl à C5 (canaux) : C1 : canal de l'Est, C2 : canal de la Marne à la Saóne, C3 : canal du Rhône au Rhin, C4 : canal de Bourgogne et $\mathrm{C}_{5}$ : canal du Centre-Chiffres cerclés : position des 13 stations de prélèvement. 


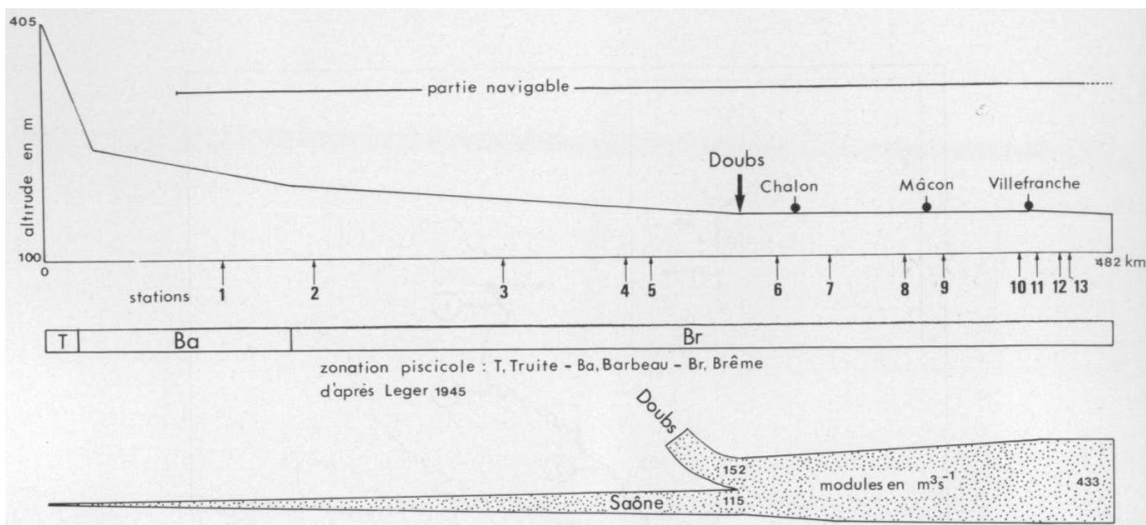

Fig. 2. Profil altitudinal - position longitudinale des 13 stations - zonation piscicole d'après Léger, 1945 - profil longitudinal des débits moyens de la Saône et du Doubs.

sont caillouteux et ceux de la Saône gravelo-sableux avec cependant quelques cailloux.

\section{HYDROLOGIE}

La Saône présente typiquement un régime de type pluvial (le Doubs, au début du printemps, apportant une légere composante nivale) avec des hautes eaux d'hiver et des étiages estivaux (Pardé 1925). Son module à Lyon est de $433 \mathrm{~m}^{3} \cdot \mathrm{s}^{-1}$, mais il n'est que de $115 \mathrm{~m}^{3} \cdot \mathrm{s}^{-1}$ à Auxonne contre $152 \mathrm{~m}^{3} \cdot \mathrm{s}^{-1}$ pour le Doubs à Neublans (fig. 2). L'étiage de la Saône, long et précoce, contraste avec celui du Haut-Rhône, court et tardif, la fonte des neiges en altitude assurant à ce dernier des débits importants en début d'été (fig. 5).

L'année 1983 constitua, hydrologiquement, pour la Saóne une année exceptionnelle avec deux crues successives : l'une normale en fin d'hiver et l'autre exceptionnelle de printemps avec, dans ce dernier cas, un débit à Couzon de plus de $2400 \mathrm{~m}^{3} \cdot \mathrm{s}^{-1}$. Au moment des crues, les variations de débit sont plus rapides dans le Haut-Rhône que dans la Saône (fig. 6).

\section{TEMPERATURE}

La Saône présente des écarts thermiques plus importants que ceux du Haut-Rhône avec des minimums hivernaux plus accusés (la Saône est susceptible de geler comme ce fut le cas, par exemple, en janvier 19.85) et des maximums estivaux plus élevés que pour le Rhône (fig. 7).

\section{AUTRES VARIABLES PHYSICOCHIMIQUES}

Dans le cadre d'une étude sur la qualité des eaux de la Saône (Agence de Bassin Rhône-MéditerranéeCorse 1984), le CemaGref a effectué une série d'analyses physico-chimiques entre le 20 et le $22 \mathrm{sep}$ tembre 1982 (débit : $75 \mathrm{~m}^{3} . \mathrm{s}^{-1}$ ) qui complètent et actualisent les données présentées par Leynaud et al. (1972). Les chlorures sont les seuls éléments qui présentent des variations significatives (tous les autres paramètres physico-chimiques mesurés montrent des variations trop irrégulières pour qu"il soit possible de mettre en évidence une tendance quel. conque). L'apport de chlorures dans la Saóne est dû aux usines SOLvaY dont les rejets contiennent jusqu'à $2525 \mathrm{mg.1}^{1.1}$ de chlorures (Agence de Bassin Rhône-Méditerranée-Corse 1984). Le Doubs, dont les eaux ne contiennent que $11 \mathrm{mg} .1^{1.1}$ de chlorures, assure une certaines dilution après la confluence, mais les teneurs restent cependant de 5 à 6 fois supérieures à ce qu'elles étaient avant le rejet (fig. 8 ). 

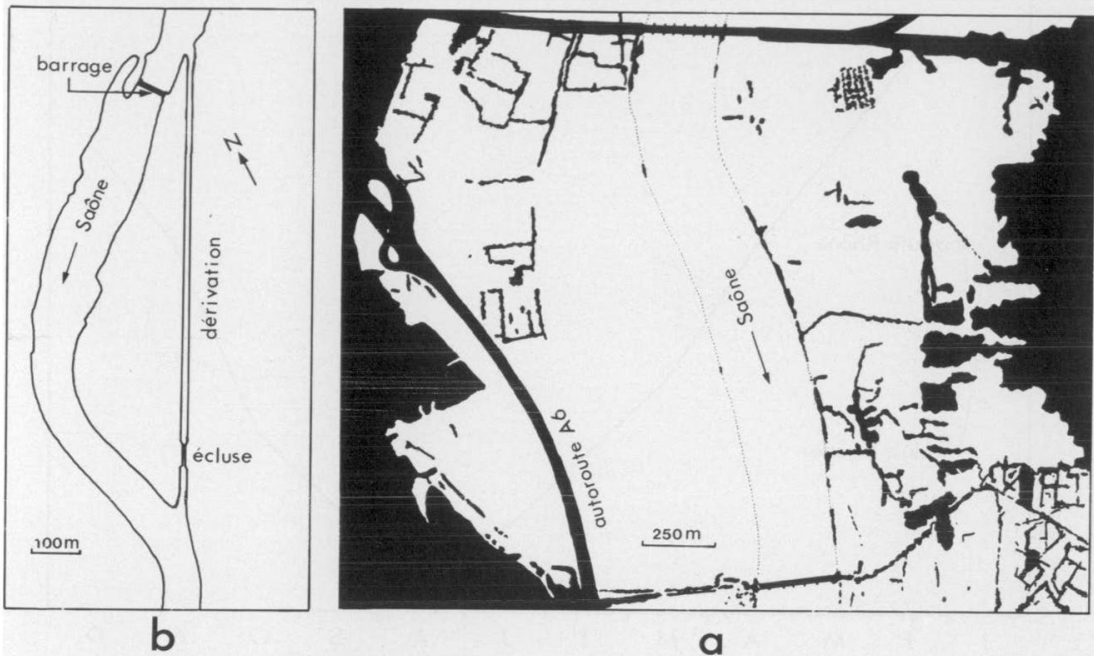

Fig. 3. a-Champ d'inondation à l'aval de Mâcon (crue de Décembre 1981, débit : $2400 \mathrm{~m}^{3} . \mathrm{s}^{1}$ ), en noir les parties émergées, en pointillés le lit mineur de la Saône. b. Un exemple de méandre court-circurité pour les besoins de la navigation : la dérivation de Rigny. Dessins d'après des clichés IGN.

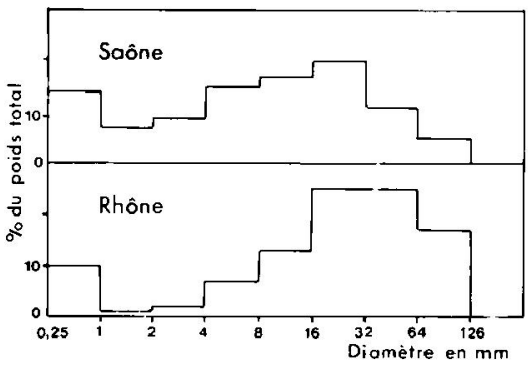

Fig. 4. Histogramme granulométrique des sédiments du chenal du Haut-Rhône à Jons et de la Saône à Beauregard.
Excepté en période de hautes eaux et au début des crues, les eaux de la Saône sont faiblement turbiL.'s: M.E.S. $=10 \mathrm{mg}^{-1}$ en moyenne (Agence de |Bassin Rhône-Méditerranée-Corse 1984)) pour la Saône, contre $7 \mathrm{mg} \cdot \mathbf{1}^{11}$ pour le Haut-Rhône (Perrin 1978).

\section{Matériels et méthodes}

Des dragages ont été réalisés entre le 2 et le 6 août 1983 avec une drague ronde de type Railler du Baty pesant $25 \mathrm{~kg}$ (diamètre d'ouverture $32 \mathrm{~cm}$ ). Ils ont été effectués au niveau de 13 stations (fíg. 1 et 2) avec deux prélèvements dans le chenal, à environ $20 \mathrm{~m}$ de la berge, l'un du cóté de la rive droite et l'autre du côté de la rive gauche.

Sur le terrain, les éléments les plus grossiers (granulométrie supérieure à $32 \mathrm{~mm}$ ) sont brossés et 


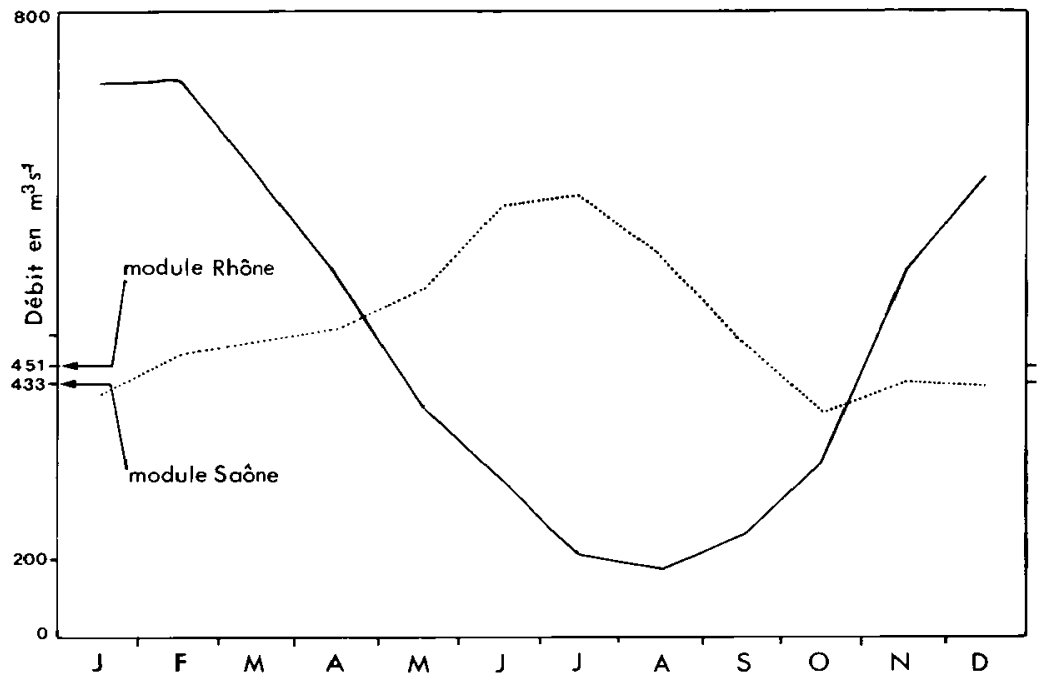

Fig. 5. Débits mensuels moyens de la Saóne à Couzon (trait plein) et du Haut-Rhône (pointillès) à Sault-Brenaz pour la période 1920-1983. D'aprés les données de la Compagnie Nationale du Rhône.

rejetés, le reste du prélèvement est versé dans une boîte étanche et fixé avec du formol à $10 \%$. Chaque dragage est précédé d'un transect de profondeur effectué à l'échosondeur de manière à déterminer la profondeur du prélèvement, la morphologie du fond et la nature du substrat (meuble ou dur).

Au laboratoire le contenu des boîtes est tamisé sur tamis de $500 \mu \mathrm{m}$, les animaux sont triés, déterminés et dénombrés par prélèvement. Nous n'avons pris en compte dans cette étude que les groupes faunistiques pour lesquels une détermination à un niveau spécifique (condition indispensable pour comparer ces peuplements avec ceux d'autres cours d'eau) était possible, c'est-à-dire les groupes suivants : Turbellariés, Achètes, Mollusques, Crustacés, Ephéméroptères, Plécoptères, Odonates, Coléoptères, Mégaloptères, Planipennes et Trichoptères. Les Dreissènes, abondantes dans de nombreux prélèvements, ont été systématiquement mesurées de manière à mettre en évidence d'éventuelles variations dans la structure des populations en place. La fraction granulométrique qui a été conservée (dont la granulométrie est comprise entre 0,5 et $32 \mathrm{~mm}$ ) a été tamisée sur une série de tamis et chaquc fraction pesée.

\section{Résultats}

\subsection{Distribution longitudinale du macrobenthos}

Un total de 47 espèces a été récolté. La répartition de ces 47 espèces dans les 26 prélèvements (fig. 9) montre une faible variabilité intrastationnelle, excepté cependant pour les prélèvements de la station 1, et une forte variabilité interstationnelle particulièrement a partir de la station 10. Les peuplements des stations 2,10 et 13 sont pauvres en espèces. Les stations 3,4 et 5 sont les plus riches. Le prélèvement le plus riche (station 3 ) ne renferme que $36 \%$ des espèces récoltées sur l'ensemble des stations. 
Année 1983

Débił maximum Saône : $2404 \mathrm{~m}^{3} \mathrm{~s}^{-1}$

Rhône : 1562

Débit minimum Saône: 55

Rhône : 182

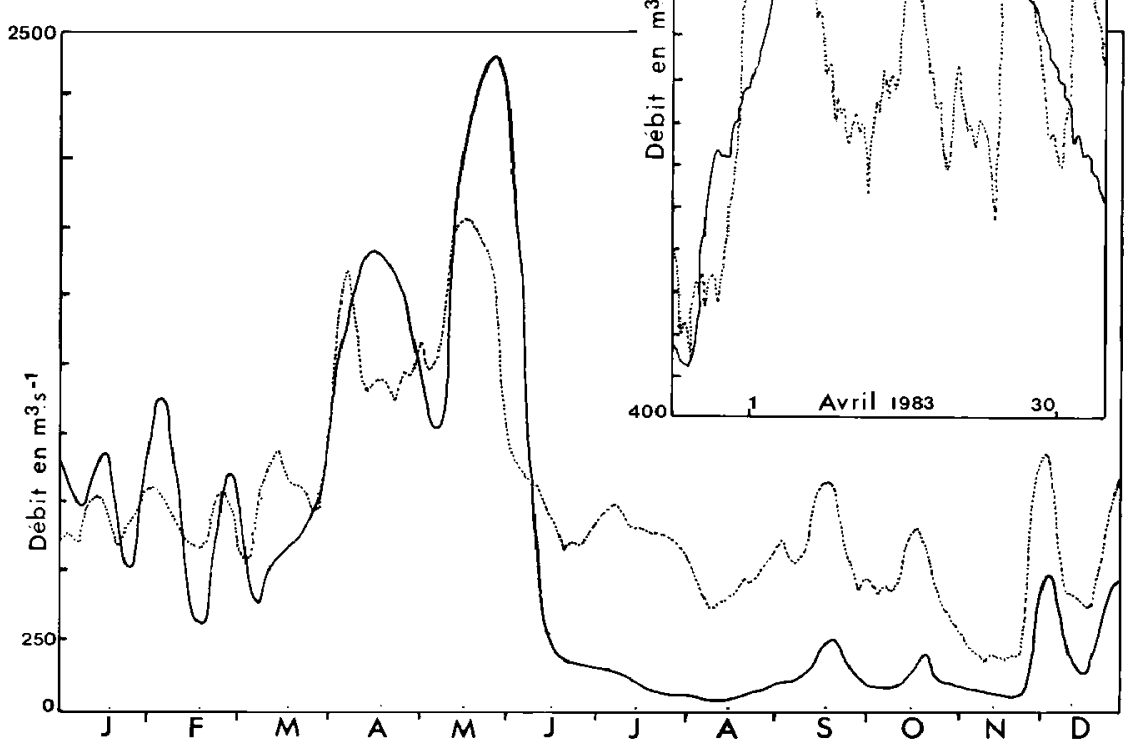

Fig. 6. Débits comparés de la Saône à Couzon (trait plein) et du Haut-Rhône (pointillés) à Sault-Brenaz pour l'année 1983. Lissage des courbes sur 8 jours. Encadré : débits journaliers de la Saône (trait plein) et du Rhône (pointillés) entre fin mars et début mai 1983. 


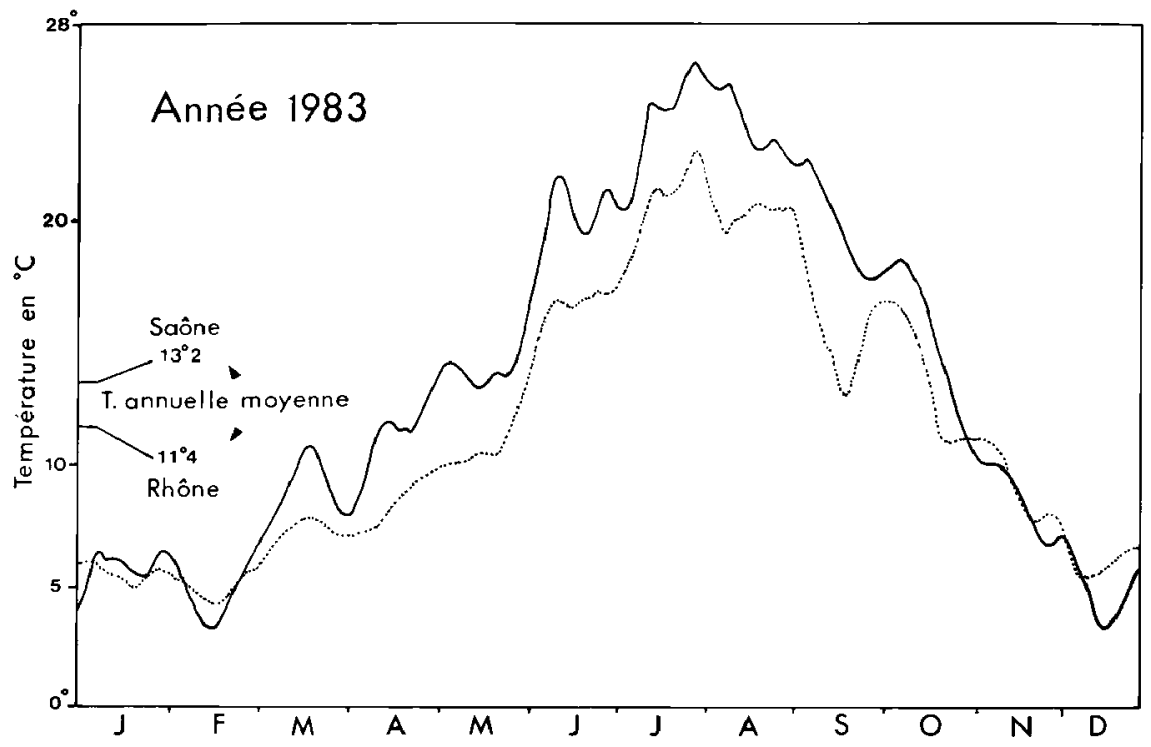

Fig. 7. Evolution comparée des températures de la Saône à Couzon (trait plein) el du Haut-Rhône (pointillés) à Sault-Brenaz en 1983. Lissage des courbes sur 8 jours. D'après les données du Service de la Navigation.

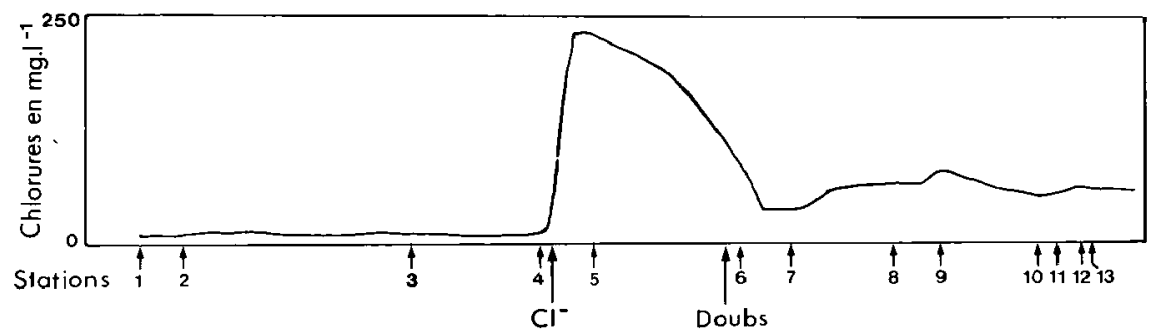

Fig. 8. Teneurs en chlorures dans la Saône (en mg. $1^{1}$ ) d'après les données de l'Agence de Bassin.

De 1 à 13 : numéro des stations. Les flèches indiquent le point de rejet des Ets Solvay (Cl') et l'arrivée du Doubs. 


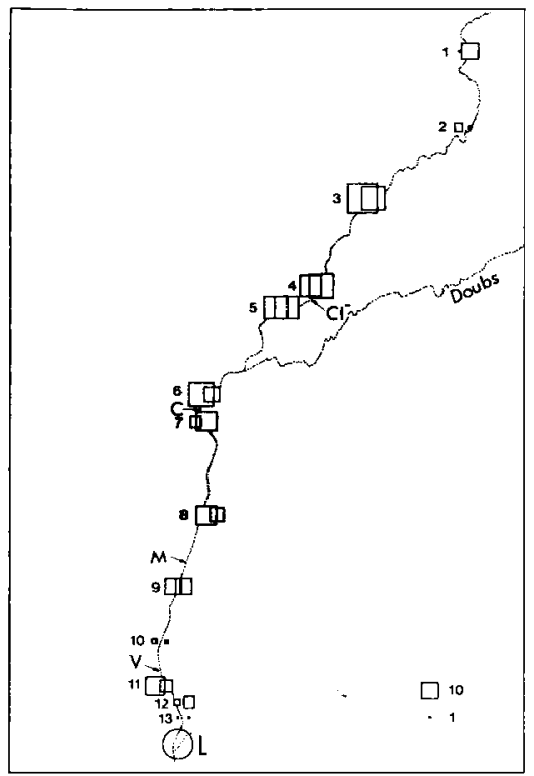

Fig. 9. Nombre d'espèces récoltées au niveau de chacune des 13 stations (deux prélevements par station). La taille des carrés est proportionnelle au nombre d'espèces par prélèvement. En pointillés le cours de la Saône et du Doubs. $\mathrm{C}$ : Chalon, $\mathrm{Cl}^{-}$: rejet de chlorures, $\mathrm{L}$ : Lyon, M : Mâcon, V : Villefranche s/Saône.

Le tableau ( 25 prélèvements $\times 46$ espèces) a été soumis à une Analyse Factorielle des Correspondances (AFC) de manière à mettre en évidence d'éventuelles structures et d'en définir la signification écologique (un prélèvement de la station 1 ne renfermant qu'une seule espèce n'a pas été inclus dans cette analyse).

Le taux d'inertie exprimé par les premiers facteurs est peu élevé (F1 : 16,9\%, F2: 12,9\%, F3 : $11,6 \%$ et $\mathrm{F} 4: 9 \%$ ) indiquant une matrice faiblement diagonalisée et donc une distribution relativement homogène des espèces. Le facteur 1 sépare nettement les 2 prélèvements de la station 10 (les seuls à contenir Lithoglyphus naticoides et une abondante population de Bythinia tentaculata) des autres prélèvements. Le facteur 3 sépare 2 prélèvements (stations 1 et 2) où se retrouvent Gomphus sp. et de nombreux Cyrmus trimaculatus, cette dernière espèce étant plus rare dans les stations aval. Les facteurs 1 et 3 , comme les facteurs 4,5 , etc... isolent ainsi un ou deux prélèvements, seul le facteur 2 oppose des groupes de stations les unes avec des coordonnées positives (stations 3, 4 et 8), les aut res avec des coordonnées négatives (stations $1,6,7,9,11$ et 12) metlant ainsi en évidence des différences moins ponctuelles ( $f$ ig. 10).

A cette différence de distribution des stations ou des prélèvements sur le facteur 2, correspond, par definition, des profils faunistiques différents. Les espèces les plus contributives pour le groupe des stalions 3,4 et 8 sont, par ordre de contribution decroissante : Pisidium supinum, Ephemera lineata, Ephoron virgo et Caenis luctuosa et pour les stations 1, 6, 7, 9, 11 et 12 : Helobdella stagnalis, Erpobdella octoculata, Asellus aquaticus et Glossiphonia complanata (fig. 10). On notera la dominance de formes fouisseuses et faiblement tolérantes dans le premier cis et d'espèces épibenthiques et plutôt tolérantes diuns le second cas.

L'étude des populations de Dreissènes (fig. 11) ne révèle de différences que pour des stations 11 et 12 où les populations sont constituées d'individus légèrement plus petits. A chaque station, la taille des individus est comprise entre 3 et $26 \mathrm{~mm}$, la taille moyenne entre 10 et $14 \mathrm{~mm}$. Si l'on se réfère aux courbes présentées par Stanczykowska (1977), les populations en place sont jeunes (environ 1 an), quelques individus ont entre 2 et 3 ans et pratiquement aucun individu n'a plus de 3 ans.

Les résultats qui viennent d'être présentés ne sont que l'expression d'une analyse objective des données. Il importe maintenant de rechercher si les structures mises en évidence par l'AFC ou la structure des populations de Dreissènes sont corrélées avec certaines variables mésologiques présentant une discontinuité longitudinale suffisamment importante pour modifier ces peuplements.

La confluence avec le Doubs, affluent dont le module est plus important que celui de la Saône (fig. 2) et dont les peuplements sont différents (Lanet 1985), pourrait expliquer les différences de composition faunistique mises en évidence par le facteur 2. 


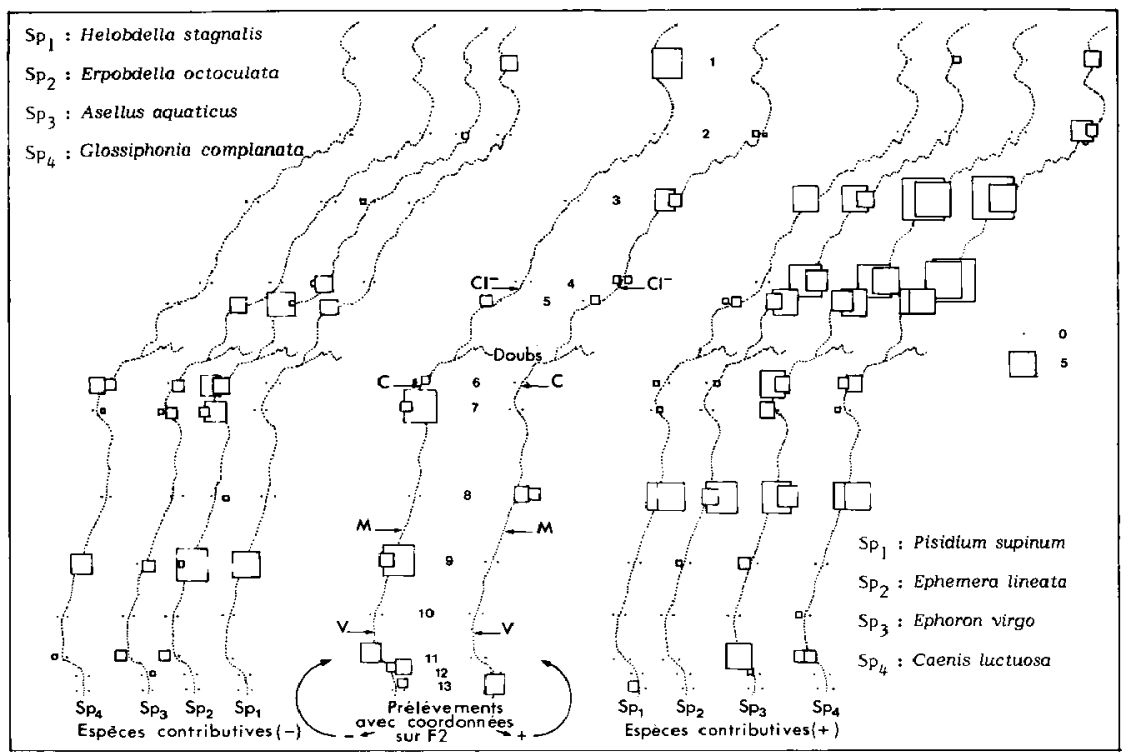

Fig. 10. Distribution des espèces les plus contributives du facteur 2 de l'AFC.

Partie centrale de la figure : projection sur une carte de la Saône des valeurs des coordonnées factorielles (taille des carrés proportionnelle à la valeur de la coordonnée) sur F2 de chaque prélèvement (deux par station, sauf station 1), - : prélèvements avec des coordonnées négatives sur $F 2$ et + : prélèvements avec des coordonnées positives sur F2. Partie gauche de la figure: Sp1, Sp2, Sp3 \& Sp4 : distribution des 4 espèces les plus contributives des prélevernents avec des coordonnées négatives sur $\mathbf{F}$. Taille des carrés proportionnelle au nombre d'individus (classes d'abondance logarithmiques de raison 2) de chacune des espèces dans le prélevement.

Partie droite de la figure: $\mathrm{Spl}, \mathrm{Sp} 2, \mathrm{Sp} 3$ \& $\mathrm{Sp} 4$ : distribution des 4 espèces les plus contributives des prélèvements avec des coordonnées positives sur $\mathrm{F} 2$. Taille des carrés proportionnelle au nombre d'individus (classes d'abondance logarithmiques de raison 2) de chacune des espèces dans le prélèvement.

Nombre de 1 à 13 : stations $-\mathrm{C}:$ Chalon, $\mathrm{Cl}^{-}$: rejet de chloru res, M : Mâcon, V : Villefranche s/Saône.

Il est évident que les structures des communautés benthiques (fig. 10), comme celle des populations de Dreissènes (fig. 11) ne sont pas modifiées après la confluence avec le Doubs.

Les teneurs en chlorures, ( $f$ ig. 8 ) très élevées à partir de la station 5 , coïncident effectivement avec l'apparition de formes tolérantes, sans qu'il y ait toutefois disparition simultanée des espèces fouisseuses (fig. 10), mais la composition faunistique de la sta. tion 8, semblable à celle des stations situées en amont du rejet, indique que l'augmentation de la teneur en chlorures a des effets peu durables. On note par ajlleurs une altération des communautés benthiques, caractérisée par la régression ou la disparition des espèces fouisseuses et l'apparition massive de formes tolérantes, à l'aval des deux principales agglomérations : Chalon et Mâcon.

La granulométrie du fond, mesurée à chaque station, ne montre pas de différence significative avec celle présentée sur la figure 4 . Les sédiments sont 


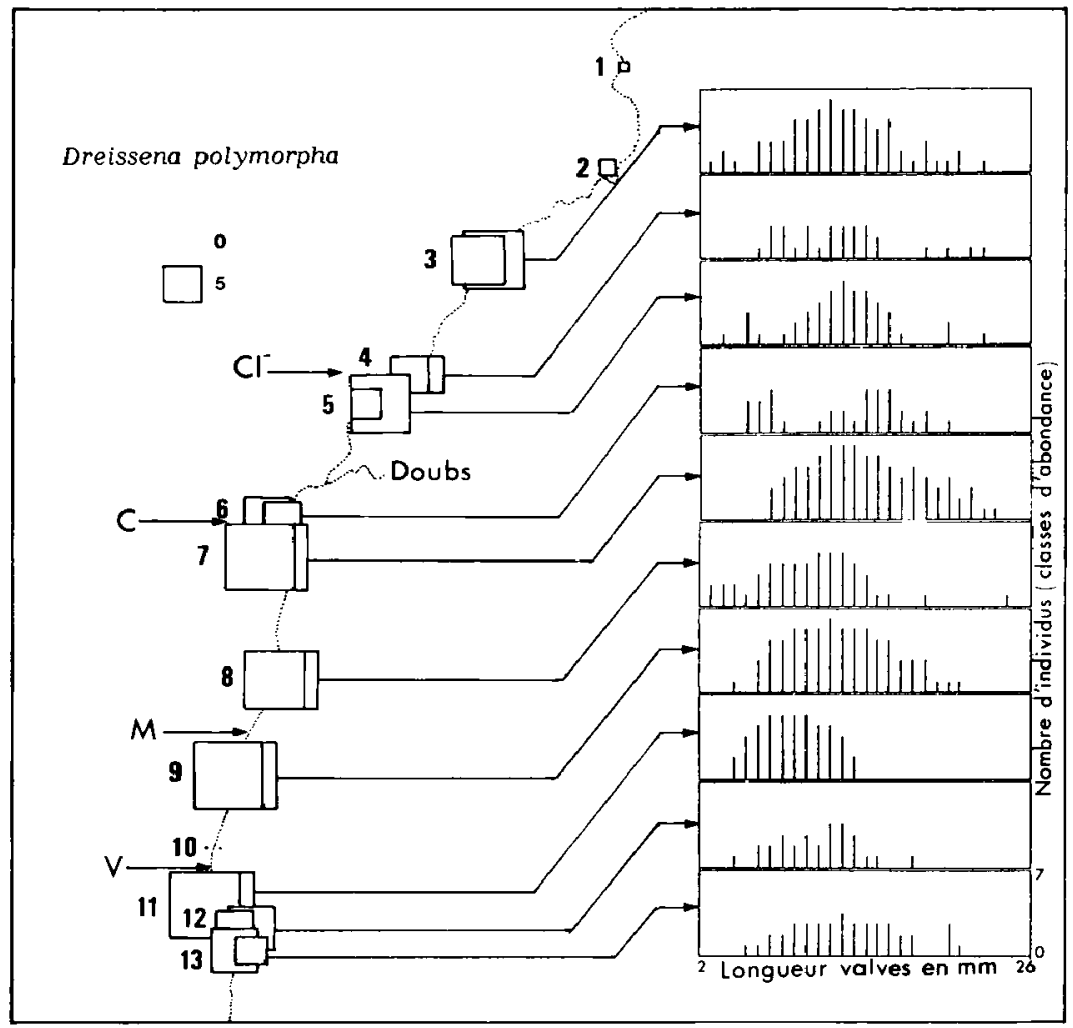

Fig. 11. Distribution de Dreissena polymorpha dans la Saône.

Taille des carrés proportionnelle au nombre d'individus (classes d'abondance logarithmiques de raison 2) dans chaque prélèvement et histogrammes de distribution des longueurs des valves à chaque station, le nombre d'individus pour chaque classe de taille étant exprimé en classes d'abondance logarithmiques de raison 2.

Nombre de 1 à 13 : stations - $\mathbf{C}$ : Chalon, $\mathrm{Cl}^{-}$: rejet de chlorures, $\mathbf{M}:$ Mâcon, $\mathrm{V}$ : Villefranche s/Saône. 
gravelo-sableux (la présence des Dreissènes sur des fonds aussi meubles s'explique par l'existence de quelques cailloux sur le fond ( $f$ ig. 4 ) sur lesquels ces Bivalves peuvent se fixer). L'absence d'espèces fouisseuses à certaines stations ne peut donc être corrélée avec des différences de composition granulométrique.

En définitive, les différents niveaux d'analyse retenus ici : macroinvertébrés et populations de Dreissènes, révèlent des modifications ou perturbations locales, mais ne mettent en évidence aucune modification longitudinale de grande ampleur dans la structure des peuplements qui présentent une grande homogénéité longitudinale en dépit d'incontestables altérations locales.

\subsection{Typologie du macrobenthos de la Saône}

La Saône présente, au moins au niveau des dimensions (largeur et profondeur) des caractéristiques voisines de celles du Haut-Rhône. Il était de ce fait intéressant de comparer la structure des communautés benthiques de ces deux cours d'eau. Comme précédemment nous n'avons pris en compte dans cette étude que les groupes faunistiques pour lesquels une détermination à un niveau spécifique (condition indispensable pour comparer les peuplements et définir une typologie) était possible, c'està-dire les mêmes groupes que dans l'étude précédente. Nous avons comparé globalement les 26 prélèvements effectués dans la Saône (qui comme nous avons pu le voir, sont relativement homogènes, au moins en termes de présence-absence) avec une série de 27 prélèvements effectués en juillet et septembre 1982 avec la même drague, dans le Rhône, à envjron $20 \mathrm{~km}$ à l'amont de Lyon par 2,5 à 7 mètres de profondeur (Gaschignard \& Berly 1987). Dans les conditions qui viennent d'être définies : à la fois systématiques (sélection de groupes de macroinvertébrés), méthodologiques (dragage) et mésologiques (macrobenthos du chenal), nous avons récolté 62 espèces dans le Haut-Rhône contre 47 dans la Saône. Sur un total de 88 espèces, seules 21 espèces sont communes aux deux cours d'eau (Tableau I).

Si l'on se réfère au système de zonation d'Illies et Botosaneanu (1963) on peut considérer le HautRhône comme un épipotamon et la Saône comme un métapotamon. A ces différences de niveau typologique correspond, comme le soulignait Cummins
(1973) dont les concepts ont été en partic repris par les promoteurs du River Continuum Concept (Vannote et al. 1980 ; Minshall et al. 1985) une structure fonctionnelle. Etant donné les difficultés que l'on peut rencontrer pour définir précisément la biologie de chaque espèce, nous n'avons considéré que trois niveaux : cycle vital, relation avec le substrat et alimentation (Tableau II).

Cycle vital : La proportion d'espèces à cycle exclusivement aquatique (par exemple Mollusques et Crustacés) est plus élevée dans la Saône que dans le Haut-Rhône, inversement les Insectes holométaboles (en particulier les Trichoptères) sont plus abondants dans le Haut-Rhône, alors que la proportion d'Insectes hémimétaboles est sensiblement la même dans les deux cours d'eau.

Relation avec le substrat : les fouisseurs sont évidemment plus nombreux dans la Saône dont les fonds présentent une granulométrie plus fine que dans le Haut-Rhône. La proportion d'espèces " sédentaires " (c'est-à-dire qui occupent une position fixe pendant la plus grande partie de leur existence comme les Dreissènes ou les Hydropsychidae par exemple) est plus élevée dans le Haut-Rhône que dans la Saône.

Alimentation : La proportion d'espèces prédatrices est plus élevée dans la Saône que dans le HautRhône. Cet te faible proportion de prédateurs dans ce dernier est sans doute due au fait que le stress hydraulique (Statzner \& Higler 1986) est plus intense dans le Haut-Rhône (Bournaud et al. 1987) que dans la Saône, empêchant ainsi la mise en place d'un réseau trophique normal. La proportion de filtreurs est sensiblement identique, même si les espèces qui assurent cette fonction ne sont pas les mêmes (comme par exemple les Pisidium), dans les deux cours d'eau.

\section{Discussion et conclusions}

La composition faunistique et la structure fonctionnelle de ces deux cours d'eau apparaissent clairement différentes, la Saône se situant, dans cette zonation longitudinale, paradoxalement plus à l'aval que le Haut-Rhône. Plusieurs facteurs mésologiques fondamentaux sont susceptibles de structurer ces peuplements, ce sont le débit, la température et la pente. 
Tăbleau I. Liste des espèces récoltées dans le Rhône et la Saône et nombre de dragages oủ l'espèce est présente. Les espèces marquées d'un $\left(^{\star}\right)$ sont communes aux deux cours d'eau.

Rhône

27 dragages
Saône

26 dragages

Turbellariés

* Dugesia tigrina

* Dugesia autres espèces

25

23

Polycelis sp.

Dendrocoelum lacteum

8

14

Mollusques

Prosobranches

* Bithynia tentaculata Lithoglyphus naticoides

* Potamopyrgus jenkinsi

* Theodoxus fluviatilis

Valvata cristata

Pulmonés

Viviparus viviparus

Ferissia

Lymnaea gr. peregra-auricularia

Gyraulus albus

Bivalves

Pisidium casertanum

Pisidium henslowanum

Pisidium subtruncatum

Pisidium moitessierianum

Pisidium supinum

* Sphaerium comeum

Sphaerium solidum

Anodonta cygnaea

Unio crassus

Unio pictorum

* Dreissena polymorpha

Crustacés

* Asellus aquaticus

Proasellus meridianus

Echinogammarus berilloni

* Gammarus fossarum

* Gammarus pulex

Gammarus roeseli

Atyaephyra desmarestii

Orconectes limosus

Ephéméroptères

Ephemera lineata

Ephemera danica

Ephoron virgo

* Heptagenia sulphurea

Heptagenia coerulans

* Ecdyonurus sp.

Rhithrogena diaphana

* Caenis luctuosa

Baetis fuscatus

Centroptilum pennulatum

Ephemerella ignita
17

1
18

14

3

5

2
8

I

9

4

13

3

9

6

2

2

1

2

1

1

5

1

3

10

3

22

3

27

27

7

3

9

3

3

9

2

2

11

1

26

9

9

3

3

23
2

8

1

1

4

2 
Rhône

27 dragages
Saône

26 dragages

Plécoptères

Leuctra gr. fusca

10

* Leuctra geniculata

Chloroperla tripunctata

Nemoura sp.

Odonates

Gomphus sp.

Coléoptères

Orectochilus villosus

Haliplus sp.

Brychius elevatus

Dryops sp.

* Elmis aenea

Elmis maugetii

Oulimnius of major

Esolus pygmaeus

Esolus parallelipipedus

Limnius volkmari

Macronychus it -tuberculatus

Stenelmis canaliculato

Riolus subviolaceus

Plannipennes

* Sysira sp.

Mégaloptères

Sialis fuliginosa

* Hydropsyche contubernalis

Hydropsyche modesta

Hydropsyche ornatula

Hydropsyche pellucidula

Hydropsyche siltalai

Polycentropus flavomaculatus

Cyrnus trimaculatus

Ecnomus tenellus

Lype phaeopa

Lype reducta

Psychomyia pusilla

* Ceraclea dissimilis

Ceraclea autres espèces.

Athripsodes albifrons

Athripsodes autres espèces.

Oecet is notata

Setodes punctatus
1

1

1

3

5

1

1

7

2

18

4

1

1

1

1

I

25

6

16

27

1

I

1

2

I

9

6

2

1

6

16

1

2

24

19

II

11

2

2 
Tableau II. Structure fonctionnelle comparée des peuplements macrobenthiques du Haut-Rhône et de la Saóne. Nombre d'espèces exprimé sous forme d'un pourcentage pour chacun des 3 niveaux : 1 - Cycle vita, 2 - Relations avec le substrat, 3 - Alimentation.

I - CYCLE VITAL

Nombre d'espèces en \% Rhône Saône

Cycle entièrement aquatique Cycle avec phase
aérienne

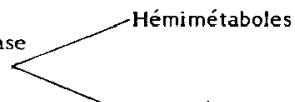
Hémimétaboles
38 60

19 20

9

17

26

\section{2 - RELATIONS AVEC LE SUBSTRAT}

$$
\text { Animaux "sédentaires" }
$$

Animaux "errants" fouisseurs

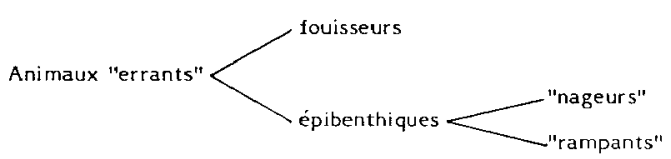

\section{7}

11

26

46
8

23

26

43

3 - AlimentATION

Dëtritivores-omnivores $<_{\text {broyeurs-racleurs }}^{\text {filtreurs }}$

Préda teurs
21

63

16
Les débits des deux cours d'eau, que l'on peut caractériser par leur module, sont très similaires (Haut-Rhône : 451 et Saône : $433 \mathrm{~m}^{3} \cdot \mathrm{s}^{-1}$ ), il ne semble donc pas y avoir de relation entre zonation longitudinale et débit. En réalité, si les débits moyens sont très semblables, le régime des deux cours d'eau est très différent (fig. 5) et, plus particulièrement, deux aspects de ce régime : d'une part la durée et l'importance de l'étiage et d'autre part les modalités de l'écoulement au moment des crues. Le nombre de mois d'étiage est plus élevé pour la Saône que pour le Haut-Rhône (fig. 5) ce qui permet la colonisation des fonds de la Saône par des espèces de milieux lénitiques, comme par exemple Ecnomus tenellus qui, comme les Polycentropodidae, tisse un filet sur le fond. Au moment des crues, par exemple celle de Mai 1983 (fig. 6), ces populations d'Ecnomidae ne sont pas détruites parce que, la Saône s'étalant largement dans son lit majeur(fig. 3a), une fraction seulement des $2400 \mathrm{~m}^{3} \cdot \mathrm{s}^{-1}$ en transit emprunte le lit mineur. Dans le Haut-Rhône, par contre, le flux correspondant à une crue analogue passe presque en totalité dans le lit mineur, entraînant un intense débit solide et la destruction des communautés macrobenthiques installées dans le chenal (Cellot et al. 1984). 
La Saóne présente une température moyenne et des écarts thermiques plus importants que le HautRhône (fig. 7) et l'on pourrait penser que la présence, par exemple d'Ephoron virgo, une especce que l'on retrouve en Espagne et en Afrique du Nord, soit corrélée avec ces températures estivales élevées et ces écarts thermiques importants. Sans exclure totalement cette hypothèse, on peut également supposer que l'absence de ce genre dans le Haut-Rhône est liée à la nature des sédiments (caillouteux) plutôt qu'à la température. Par ailleurs, la présence dans les deux cours d'eau d'une espece thermophile: comme Dugesia tigrina, laisse supposer que la température ne joue qu'un faible rôle dans la structuration des communautés benthiques ce qui confirme les observations de Statzner \& Higler (1986).

La pente des deux cours d'eau est très différente (cf. $\$ 2.1$.), elle conditionne à la fois le courant et la granulometrie et c'est sans doute elle qui, avec les différences de régime, joue un rôle essentiel pour définir la composition de ces communautés et expliquer ainsi pourquoi la Saône correspond à un métapotamon et le Haut-Rhòne à un épipotamon.

La plupart des cours d'eau appartenant à un niveau typologique analogue sont actuellement, au moins dans l'hémisphère Nord, profondément dégradés (Fittkau \& Reiss 1983) et on peut se demander, dans le cas de la Saône, ce qui subsiste du peuplement benthique originel. En l'absence de relevés anciens, nous ne pouvons que comparer sa situation avec celle d'autres cours d'eau présentant des caractéristiques mésologiques (et donc typologiques) similaires et pour lesquels des données sur les macroinvertébrés (déterminés au niveau spécifique) sont disponibles. C'est le cas en particulier de la Volga (Beh. ning 1928), du Rhin (Neeracher 1910, Lauterborn 1917) et de la Moselle (Mauch 1963) pour l'Europe et du Mississipi supérieur (Fremling 1960, 1964) pour l'Amérique du Nord.

Les données dont nous disposons concernent surtout les insectes et plus particulièrement les Ephéméroptères, les Plécoptères et les Trichoptères.

Les fonds de ces grands cours d'eau étant constitués de sables ou d'argiles, les espèces fouisseuses d'Ephéméroptères (en particulier Ephoron virgo), dont les larves se rencontraient en abondance au début du siècle dans le cours inférieur de la Volga (Behning, 1928) et le cours moyen du Rhin (Neeracher 1910, Lauterborn 1917), font partie de ce peuplement originel. Elles ont disparues du Rhin (Fittkau \& Reiss 1983) et sont maintenant rares dans la Moselle (Mauch 1963). En Amérique du Nord, il existait dans le Mississipi supérieur (Fremling 1964) d'abondantes populations d'Ephéméroptères fouis. seurs (Hexagenia bilineara, $H$. limbata el Penagenia vittige ra). Leurs populations paraissent avoir fortement régressé depuis cet te période (Elstad 1986). La Saône, qui hébergeait d'importantes populations d'Ephoron virgo et d'Ephemera lineata (ValentinSmith 1852, Denis et al. 1936, Fontaine 1959) a apparemment conservé son peuplement originel d'Ephéméroptères fouisseurs, même si leurs populations sont à la fois moins abondantes et plus localisées.

Il y a peu d'espèces de Plécoptères dans des cours d'eau du type de la Saône. Il s'agit, pour l'Europe, soit de Leuctridae (en particulier Letictra gr. fusca et Leuctra geniculata) ou de Perlidae et notamment Marthamea selysii que Mauch (1963) récolte, avec une certaine constance, dans la Moselle. Excepté Leuctra geniculata, nous n'avons pas trouvé d'autres Plécoptères dans la Saône, mais l'absence de Marthamea selysii (ou celle de $M$. vitripennis) est peutètre due à la nature des fonds, ceux de la Moselle sont en effet légèrement plus grossiers que ceux de la Saóne. Dans le Mississipi supérieur, une seule espèce de Plécoptères (Perlesta placida, un Perlidae à large distribution longitudinale), a été trouvée (Elstad 1986).

Les fonds étant meubles, les Trichoptères sont peu représentés dans le benthos du chenal. Ce sont essentiellement des Hydropsychidae, des Polycentropodidae et des Ecnomidae. Le petit nombre d'Hydropsyche contubemalis trouvé dans la Saône ne tient pas à un phénomène de pollution, puisque le Rhin, plus pollué chimiquement que la Saóne, héberge, de nouveau, d'abondantes populations d' $H$. contubernalis, (Higler \& Tolkamp 1983), mais résulte du caractère fortement lénitique de cette rivière. Bournaud et al. (1987) ont en effet mont ré que dans le Haut-Rhòne, un étiage précoce et prolongé entraine une reduction des populations d'Hydropsy. chidae. L'abondance des Cymus irimaculatus dans les stations amont, puis d'Ecnomus tenellus dans les stations aval (fig. 12) indique également des courants très faibles puisque la deuxième espèce se rencontre également cn cau stagnante. E. tenellus est également abondant dans la Meuse (Mauch 1963). Dans le Mississipi supérieur, Fremling (1960) trouve trois espèces d'Hydropsychidae (Hydropsyche orris, 


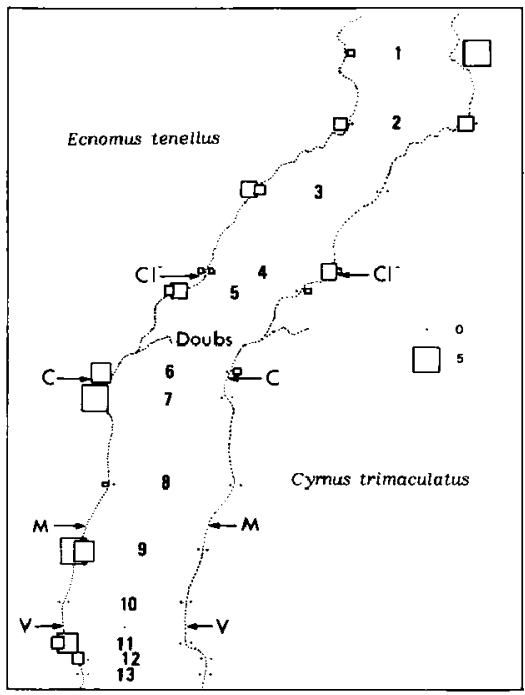

Fig. 12. Distribution des larves de Cymus trimaculatus ct d'Ecnomus tenellus dans la Saóne. Taille des carrés proportionnelle au nombre d'individus (classes d'abondance logarithmiques de raison 2) de chacune des espèces dans le prélèvement.

Nombre de 1 à 13 : stations - $\mathrm{C}$ : Chalon, $\mathrm{Cl}^{-}:$rejet de chlorures, $\mathbf{M}$ : Mâcon, $\mathbf{V}:$ Villefranche s'Saóne.

Cheumatopsyche campyla et Potamyia flava), ce qui laisse supposer des courants plus élevés que dans la Saône, et un Polycentropodidae (Cymellus marginalis). Cette dernière espèce occupant sans doute la niche que Cymus trimaculatus et Ecnomus tenellis tiennent en Europe.

L'arrivée d'espèces nouvelles dans la Saône, comme par exemple Dreissena polymorpha, Potamopyrgus jenkinsi ou Gammarus roeseli, de même que la dégradation des populations piscicoles (Léger 1945. Souchon 1981) lièe en particulier aux aménagements du Bas-Rhône ont dû altérer un édifice trophique particulièrement instable puisque fonctionnant en milieu lénitique et donc modifier ainsi la structure originelle des communautés benthiques.
Cependant, l'impossibilité pour l'Homme de s'installer de façon permanente dans les zones inondables a limité les effets destructeurs d'une anthropisation excessive quj caractérise de nombreux grands cours d'eau européens ou Nord américains. Ceci explique que, dans certains secteurs, la Saône présente encore certains éléments des communautés benthiques originelles d'une grande rivière de plaine, dont la lenteur, comme le soulignait Caesar, est effectivement exceptionnelle.

\section{Remęrçemęntș}

Les auteurs remercient la Compagnie Nationale du Rhône et le Service de la Navigation qui leur ont permis d'utiliser les données concernant les débits et températures. Ils remercient également Mme M. Richardot-Coulet pour la détermination des Mollusques et Mrne $C$. Roux pour la détermination des Amphipodes. Les calculs et les représentations graphiques ont étè réalisés à partir de progranmes mis au point par MM. D. Chessel et $Y$. Auda du laboratoire de Biométrie de Lyon I.

\section{Travaux cités}

Agence de Bassin Rhóne-Méditerranée-Corse. 1984. - Etude de la pollution de la Saóne. Rapport de l'Agence: $162 \mathrm{p}$.

Behning (A.). 1928. - Das Leben der Wolga. Binnengewässer, (5) $162 \mathrm{p}$.

Boumaud (M.), Tachet (H.) \& Roux (A.L.). 1987. - The effects of seasonal and hydrological influences on the macroinvertebrates of the Rhone River. France. II - Ecological aspects. Arch. Hydrobiol., Suppl. Bd 76: 25.51.

Bourrain (X.) \& Bourrain (P.) 1983. - Essai d'appreciation de l'impact des exploitations de granulats sur la macrofaune ben. thique de quelques frayeres de Cóte d'Or et de Saône et Loire. Rapport Univ. Dijon, Lab. Biol. Anim. : 21 p.

Cellot (B.), Bournaud (M.) \& Tachet (H.). 1984 - The movements of larvae of Hydropsyche (Trichoptera) in a large river. Proc 4th Int Symp. Trichoptera Ed. Morse, Clemson, $30: 57-68$.

Cellot (B.). Maucet (D.) \& Boumaud (M.). 1987. - Applications aux grandes rivieres d'un indice de qualité biologique basé sur les macroinvertébres benthiques. Sciences de liats, 6: 473-495.

Cemagref. 1984. - Etude écologique de la Saóne entre Auxonne et Tournus - Etat de référence - Rapport Cemagref, Div. Qual. Eaux, Pêche et Pisciculture: $158 \mathrm{p}$.

Cummins (K.W.). 1973. - Trophic relations of aquatic insects Anne. Rev. Entomol. 18: 183-206.

Denis (J.R.). Paris (P.) \& Pillon (M.). 1936. - Notes sur le Polymitarcis virgo Ol, la manne blanche des riverains de la Saône. Bull. Scient. Bourgagne, 6: 99-t04.

Elstad (C.A.). 1986. - Macrobenthic distribution and community structure in the upper navigation pools of the Upper Mississipi River. Hydrobiologia, $136: 85-100$.

Fittkau (E.J.) \& Reiss (F.). 1983. - Versuch einer Rekonstruktion der Fauna europäischer Ströme und ihrer Auen. Arch. Hydrobiol., $97: 1.6$

Fontaine (J.) 1959. - Un problème biologique interessant : les chutes de manne. Sci. Nat., Paris, $32: 9.14$. 
Fremling (C.R.). 1960. - Biology and possible control of nuisance Caddisflies of the Upper Mississipi River. Res. Bull. Iou'a Sta. Univ. Sci. Technol., $485: 856 \cdot 879$.

Fremling (C.R.) 1964. - Mayfly distribution indicates water qua. lity on the Upper Mississipi River. Science, $146: 1164$ - 1166.

Gaschignard (O.) \& Berly (A.). 1987. - Impaci of large discharge fluctuations on the macroinvertebrate populations downstream a darn. 3rd Int. Symp. Regulated streams, Edmonton, $1985: 145-161$

Higler (L.W.G.) \& Tolkamp (H.H.). 1983. - Hydropsychidae as bior indicator. Envir. Mon. Assessm., 3 : 331-341.

Jazdzewski (K.) \& Roux (A.L.). 1987. - Biogéographie de Gumma. rus roeseli Gervais (Crusiacé Amphipode) en Europe, en particulier répartition en France el en Pologne. Crustaceana (sous presse).

Lanet (B.). 1985. - Influence des extractions de granulats sur les macroinvertébrés du Doubs inférieur. Rapport DEA, Lyon : $40 \mathrm{p}$.

Lauterborn (R.). 1917. - Die geographische und biologische Gliederung des Rheinstroms. II. Teit. S Sber. Heidelberger Acad. Wiss. Mathem. natum!, $5 ; 1.70$.

Léger (L.). 1945. - Carte piscicole du département du Rhône. Trav. Lab. Hydrobiol. Piscic. Univ. Grenoble : $16 \mathrm{p}$.

Leynaud (G.), Nisbet (M.), Rofes (G.) \& Verneaux (J.). 1972. Etude à caractère synthétique d'un grand cours d'eau de plaine : Ia Saóne. Verh. Intemat. Verein. Limnol., 18 : 706-717.

Locard (A.). 1893. - Les Dreissensia du système européen d'après la collection Bourguignat. Revie Staisse Zool., 1: 113-185.

Mauch (E.). 1963. - Untersuchungen über das Benthos der deuts. chen Mosel unter besonderer Berücksichtigung der Wasser. güte. Mitc. Zool. Mus. Berlin, $39 ; \mathbf{1 . 1 7 2}$.

Minshall (G.W.), Cummins (K.W.), Petersen (R.C.), Cushing (C.E.), Bruns (D.A.), Sedell (J.R.) \& Vannote (R.L.). 1985. Developments in Strearn Ecosystem Theory. Can. J. Fish. Aquat. Sci. $42=1045-1055$.
Neeracher (F.). 1910. - Die Insektenfauna des Rheins und seiner Zuflüsse bei Basel. Revtue siuisse Zool., 18 : 497-590.

Pacaud (A.). 1952. - Nouvelle revue de la distribut ion géographique des Gammares dans les eaux douces continentales fran. çaises. C.R. summ. Séanc. Soc. Biogéogr., 252 : 95-111.

Parcle (M.). 1925. - Le régime du Rhône (I et II). Ed. P. Masson, Lyon: 887 p, et $440 \mathrm{p}$.

Paris (P.). 1931. - Crustacés de la Saône movenne. Bull. Scient. Bourgogne, 1 ; 27.31 .

Perrin (J.F.). 1978. - Signification écologique des peuplements benthiques du Haut Rhóne français. Thèse de 3 eycle, Lyon : $171 \mathrm{P}$.

Regis (J.), Gaude (T.), Pattee (E.), Persat (H.) \& Zandonella (P.). 1980. - La Saóne à son entrée dans l'agglomération lyon. naise : étude physicochimique et biologique. Bull. Soc. Linn. Lvon, $10: 550-573$.

Souchon (Y.). 1981, - Recensernent des zones sensibles et des biotopes aquatiques remarquables de la Saóne depuis sa confluence avec le canal de l'Est jusqu'au Rhône. Rapport Conseil Supérieur de la pêche : $21 \mathrm{p}$.

Vannote (R.L.), Minshall (G.W.). Cummins (K.W.), Sedell (J.R.) \& Cushing (C.E.). 1980. - The River Continuum Concept. Can. J. Fish. Aquar. Sci., $37:$ 130-137.

Stanczykowska (A.). 1977. - Ecology of Dreissena polymorpha (Pall.) (Bivalvia) in lakes. Polsk. Arch. Hydrobiol., 24 : 461.530.

Statzner (B.) \& Higler (B.). 1986. - Stream hydraulics as a major determinant of benthic invertebrate zonation patterns Fresh. Bivl., 16, 127.139.

Valentin-Smith (J.E.). 1852. - Monographie de la Saône. Revue du Lyonnais. Impr. L. Boitel : 33-168. 